\title{
Cyclic Cohomology of Corings
}

\author{
Bahram Rangipour \\ Department of Mathematics \\ The Ohio State University \\ Columbus, $\mathrm{OH}$ 43210, USA
}

October 9, 2017

\begin{abstract}
We define cyclic cohomology of corings over not necessarily commutative algebras. We observe that being para Hopf algebroid of enveloping algebra of an algebra is the key fact which allows us to define this cohomology. This observation enables us to define Hopf cyclic cohomology of corings on which para Hopf algebroids act.
\end{abstract}

\section{Introduction}

Cyclic cohomology invented by Connes [5] as a noncommutative analogue of de Rham homology, it is a far-reaching part of noncommutative geometry which has successfully participated in many areas such as Novikov conjecture for hyperbolic groups and noncommutative index theory. Connes defines it as the cohomology of the invariant co-chains under the action of the cyclic operator which form a subcomplex of Hochschild complex [5]. Cyclic cohomology can be defined, in the most general definition, for any cocyclic object in any abelian category [6]. For a comprehensive account in cyclic (co)homology one can see [17. A cocyclic object in a category is a covariant functor from the cyclic category to that category. Cyclic cohomology as such is a very powerful cohomology theory with all good properties that a cohomology theory could have. Its applications and importance in noncommutative geometry urges us to study and develop it to new algebraic constructions. 
Hopf cyclic cohomology of Hopf algebras was defined by Connes and Moscovici for any Hopf algebra satisfying some certain properties [8]. They showed that cyclic cohomology of their Hopf algebra $\mathcal{H}_{n}$, assigned to any manifold of dimension $n$, determines the class of the index cyclic cocycle which computes the index of transversal elliptic operator on that manifold. It is shown that this cohomology is a very fit generalization of Lie algebra cohomology and group cohomology to the realm of quantum groups [8, 16]. Cyclic cohomology of algebras, Hopf cyclic cohomology of Hopf algebras, and almost all other cyclic cohomologies known so far has been unified in a single theory called Hopf cyclic cohomology with coefficients [11]. Hopf cyclic cohomology is also seen as a generalization of invariant de Rham cohomology in noncommutative geometry [15].

The role of groups in geometry as the source of symmetry is played by Hopf algebras in noncommutative geometry. The need of groupoids in classical geometry as a group with several objects repeats in noncommutative geometry to introduce Hopf algebras with several objects or Hopf algebroids. We refer the reader to $3,4,12,18,20,19,21,22,23]$ to trace the evolution of Hopf algebroids. In the first attempt to develop Hopf cyclic cohomology for Hopf algebroids, para Hopf algebroids are introduced as the suitable gadgets on which cyclic cohomology of Hopf algebras can be generalized [14]. A para Hopf algebroid is a bialgebroid endowed with anti-algebra map called twisted antipode which satisfies some certain conditions. An example of para Hopf algebroid is Connes-Moscovici algebra in non-flat case. The other examples are some special crossed product algebras such as quantum torus.

Corings can be seen as coalgebras over noncommutative algebras. Their examples are speared from the algebras of differential forms over a manifold to Hopf-Galois extensions. One can assign to any ring extension a coring which speak of the fact that coring is an algebraic object, on the other hand to any finitely generated projective module over an algebra one assigns a coring which turns to the coring of $n$ by $n$ matrices over the algebra in the case that the module is free. The latter is an example of crossed product coring. Many cohomology theories such as intertwine cohomology can be redefine in terms of cohomology of coring [1]. In this paper we develop Hochschild and cyclic cohomology of coring. We observe that the idea of Hopf-cyclic cohomology is very useful to pass the difficulty that the cyclic operator is not well-defined when the ground algebra is not commutative and/or the left and right action of ground algebra are not the same.To pass this difficulty one has to understand the real nature of Hochschild and cyclic complex of a 
coalgebra. Although for a coalgebra all the tensors are over the ground field, the role of ground field in the first tensor is different from the other tensors. In the first tensor it plays as a para Hopf algebroid and in the other tensors its role is the same as an algebra.

Although the concept of para Hopf algebroid and in general bialgebroid is not, in contrast with that of bialgebra, self-dual, we show that the dual theory of its cyclic cohomology is indeed rich and contains some algebraic information. Para Hopf algebras can be seen as a very richer source of symmetry in noncommutative geometry than Hopf algebras or quantum groups, they can act on coalgebras and algebras with several objects, which are called coring and ring in this paper respectively. Throughout this paper we always denote ground fields by $k$, ground algebras by $R$ and $S$, corings by $\mathcal{C}$ and $\mathcal{D}$, para Hopf algebroids by $H$, and a Hopf algebras by $\mathcal{H}$.

\section{Corings and para Hopf algebroids}

In this section we introduce corings and their sources of symmetry which is the category of para Hopf algebroids. They can be seen as coalgebras and Hopf algebras with several objects respectively. We give some examples which will be used in the sequel sections.

Definition 2.1. A coring over a $k$-algebra $R$ is an $(R, R)$-bimodule $\mathcal{C}$ with two $(R, R)$-bimodule maps

$$
\Delta: \mathcal{C} \rightarrow \mathcal{C} \otimes_{R} \mathcal{C}, \quad \text { and } \quad \epsilon: \mathcal{C} \rightarrow R,
$$

that are called coproduct and counit respectively, with the following properties

$\left(I d_{\mathcal{C}} \otimes_{R} \Delta\right) \circ \Delta=\left(\Delta \otimes_{R} I d_{\mathcal{C}}\right) \circ \Delta, \quad$ and $\quad\left(I d_{\mathcal{C}} \otimes_{R} \epsilon\right) \circ \Delta=I d_{\mathcal{C}}=\left(\epsilon \otimes_{R} I d_{\mathcal{C}}\right) \circ \Delta$

We use Sweedler's notation for working with the image of $\Delta$ in $\mathcal{C} \otimes_{R} \mathcal{C}$, i.e.,

for $c \in \mathcal{C}$ we write $\Delta(c)=\sum c^{(1)} \otimes_{R} c^{(2)}$, when the summation is understood. Sometimes we even omit the symbol $\sum$.

One can think of corings as a generalization of coalgebras and algebras simultaneously. If you have a $k$-coalgebra it is obvious that it is a coring over $k$ and if you have an algebra it is a coring over itself with identity map 
as coproduct and counit. We refer the reader to [1] for a comprehensive account to have more information about corings. A coring over $R$ can also be interpreted as coalgebra object in the category of $R$-module.

We would like to endow the category of coring with the most general symmetry that we can. We know that for coalgebras the most general symmetry that one can define is the action and coaction of bialgebras. For corings we have a thick version of bialgebras as symmetry which are called bialgebroid. In the following we recall the definition of bialgebroid.

Let $H$ and $R$ be two algebras with an algebra homomorphism $\alpha: R \rightarrow H$, and an algebra antihomomorphism $\beta: R \rightarrow H$ such that the images of $\alpha$ and $\beta$ commute in $H$, i.e. for all $a, b$ in $R$

$$
\alpha(a) \beta(b)=\beta(b) \alpha(a) .
$$

It follows that $H$ has an $R$-bimodule structure defined by

$$
a x b=\alpha(a) \beta(b) x \quad \forall a, b \in R, x \in H .
$$

We call $(H, R, \alpha, \beta, \Delta, \epsilon)$ a bialgebroid if

i) $(H, R, \Delta, \epsilon)$ is a coring over $R$, when $H$ is a $R$ bimodule via $\alpha$ and $\beta$.

ii) Compatibility with the product: for all $a, b \in H$ and $r \in R$,

$$
\begin{gathered}
\Delta(a)(\beta(r) \otimes 1-1 \otimes \alpha(r))=0 \quad \text { in } H \otimes_{R} H, \\
\Delta(a b)=\Delta(a) \Delta(b) .
\end{gathered}
$$

In the first relation the natural right action of $H \otimes H$ on $H \otimes_{R} H$ defined by $\left(a \otimes_{R} b\right)\left(a^{\prime} \otimes b^{\prime}\right)=a a^{\prime} \otimes_{R} b b^{\prime}$ is used. While $H \otimes_{R} H$ need not be an algebra, it can be easily checked that the left annihilator of the image of $\beta \otimes 1-1 \otimes \alpha$ is an algebra. Hence, by the first relation, the multiplicative property of $\Delta$ makes sense.

iii) The counit is unital,

$$
\epsilon(1)=1 .
$$

Definition 2.2. A bialgebroid $(H, R, \alpha, \beta, \Delta, \epsilon)$ is called a Para Hopf algebroid if there is an antialgebra map $T: H \rightarrow H$, called a para-antipode, satisfying the following conditions:

PH1) $T \beta=\alpha$. 
PH2) $m_{H}(T \otimes i d) \Delta=\beta \epsilon T: H \rightarrow H$, where $m_{H}: H \otimes H \rightarrow H$ is the multiplication map of $H$.

PH3) $T^{2}=i d_{H}$, and for all $h \in H$

$$
T\left(h^{(1)}\right)^{(1)} h^{(2)} \otimes_{R} T\left(h^{(1)}\right)^{(2)}=1 \otimes_{R} T(h) .
$$

Let $\mathcal{C}$ be a coring over an algebra $R$. We say a bialgebroid $(H, R)$ acts on $\mathcal{C}$ if $\mathcal{C}$ is a $H$-module, here $H$ is thought only as an algebra over $k$. For defining the notion of Hopf module coring we need to restrict $H$-module structure of $\mathcal{C}$ a little bit more. That is, the actions of $R$ induced by $\alpha$ and $\beta$ on $\mathcal{C}$ have to be coincident with the left and right actions of $R$ respectively. By the above assumption we can define the diagonal action of $(H, R)$ on $(\mathcal{C}, R)$ as follows

$$
\begin{gathered}
H \otimes \mathcal{C} \otimes_{R} \mathcal{C} \longrightarrow \mathcal{C} \otimes_{R} \mathcal{C} \\
h \cdot\left(c \otimes_{R} d\right)=h^{(1)} c \otimes_{R} h^{(2)} d \quad \text { for any } h \in H \text { and } c, d \in \mathcal{C} .
\end{gathered}
$$

This defines an action because of (ii) in the definition of bialgebroids. We know that the base algebra as such is a coring over itself. We want to endow it an structure of $H$-module coring. To this end, we have to assume one more axiom for bialgebroids, that is for any $g, h \in H$

$$
\epsilon(h g)=\epsilon(h \beta(\epsilon(g))) \text {. }
$$

This condition in many sources is one of the axioms of bialgebroids. One can show that for a para Hopf algebroid this condition is satisfied for free [14. Thanks to the above assumption on $\epsilon$ one defines the following action of a bialgebra $(H, R)$ on $R$.

$$
\begin{gathered}
H \otimes R \longrightarrow R \\
h \cdot r=\epsilon(h \alpha(r))=\epsilon(h \beta(r))
\end{gathered}
$$

Which is a generalization of trivial action of Hopf algebras on the ground ring.

Definition 2.3. Assume that $(H, R)$ acts on a coring $\mathcal{C}$ and this action has the above property, i.e. the left and right actions of $R$ coincide with its actions induced by the action of $H$ on $C$. We call $\mathcal{C}$ an $H$-module coring if $\Delta: \mathcal{C} \rightarrow \mathcal{C} \otimes_{R} \mathcal{C}$ and $\epsilon: \mathcal{C} \rightarrow R$ are $H$-module maps, where $\mathcal{C} \otimes_{R} \mathcal{C}$ and $R$ are $H$-module as above. 
The above definition generalizes Hopf module coalgebras. One sees that action of any bialgebroid on itself by multiplication satisfies the above definition.

Example 2.1. Let $B \rightarrow A$ be a $k$ algebra extension. The Sweedler's bialgebroid $A_{B}^{\mathrm{e}}=A \otimes_{B} A^{\mathrm{op}}$ is defined as follows.

$$
\begin{array}{llr}
\alpha: A \rightarrow A_{B}^{\mathrm{e}}, & \text { by } & a \mapsto a \otimes_{B} 1_{A} \\
\beta: A \rightarrow A_{B}^{\mathrm{e}}, & \text { by } & x \mapsto 1_{A} \otimes x \\
\Delta: A_{B}^{\mathrm{e}} \longrightarrow A_{B}^{\mathrm{e}} \otimes_{A} A_{B}^{\mathrm{e}} & \text { by } & a \otimes x \mapsto a \otimes 1_{A} \otimes_{A} 1_{A} \otimes x \\
\epsilon: A_{B}^{\mathrm{e}} \longrightarrow A & \text { by } & a \otimes x \mapsto a x
\end{array}
$$

The Sweedler's bialgebroid $R^{\mathrm{e}}=R_{k}^{\mathrm{e}}$ acts on any coring $\mathcal{C}$ over the algebra $R$ by

$$
(r \otimes s) c=r \cdot c \cdot s .
$$

One can show that in fact $\mathcal{C}$ is a $R^{\mathrm{e}}$-module coring.

Example 2.2. Any bialgebroid $(H, R)$ acts on $A^{e}$ via $h(a \otimes b)=\epsilon_{H}\left(h^{(1)} \alpha(a)\right) \otimes$ $\epsilon_{H}\left(h^{(1)} \beta(a)\right)$ and makes $A^{e}$ a left $H$-module coring.

Let $\mathcal{H}$ be a Hopf algebra with an invertible antipode. Let $M$ be a left $\mathcal{H}$-module and a right $\mathcal{H}$ comodule. It is called Yetter-Drinfeld module if

$$
\Delta_{M}(h m)=h^{(2)} m^{(0)} \otimes h^{(3)} m^{(1)} S^{-1}\left(h^{(1)}\right) .
$$

Then $\mathcal{H}^{\mathcal{H}}$, the category of Yetter-Drinfeld module over $\mathcal{H}$, consists of the above objects and usual morphisms. It forms a braided category with the following braiding map:

$$
\sigma: M \otimes N \rightarrow N \otimes M, \quad(m \otimes n) \mapsto n^{(0)} \otimes n^{(1)} m .
$$

Let $A \in \mathcal{H}^{\mathcal{H}} \mathcal{D}^{\mathcal{H}}$ be a braided commutative algebra in ${ }_{\mathcal{H}} \mathcal{Y} \mathcal{D}^{\mathcal{H}}$, i.e., it is a left $\mathcal{H}$ module algebra and right $\mathcal{H}^{\text {op }}$ comodule algebra and for any $a, b \in A$ we have

$$
b^{(0)}\left(b^{(1)} a\right)=a b
$$


It is shown in 2] that the crossed product algebra $H=A \sharp \mathcal{H}$ is a Hopf algebroid over $A$ with the following structure.

$$
\begin{array}{ll}
\Delta: H \rightarrow H \otimes_{A} H, & \Delta(a \# h)=a \# h^{(1)} \otimes_{A} 1 \# h^{(2)}, \\
\epsilon: H \rightarrow A, & \epsilon(a \# h)=a \epsilon(h), \\
\alpha: A \rightarrow H, & \alpha(a)=a \# 1, \\
\beta: A \rightarrow H, & \beta(b)=b^{(0)} \# b^{(1)}, \\
\tau: H \rightarrow H, & \tau(a \# h)=S\left(h^{(2)}\right) S^{2}\left(a^{(1)}\right) a^{(0)} \# S\left(h^{(1)}\right) S^{2}\left(a^{(2)}\right) .
\end{array}
$$

Let $S^{2}=i d$ and $A$ is stable, i.e., $a^{(1)} a^{(0)}=a$. We show that the crossed product algebra $H=A \# \mathcal{H}$ is a coring over $A$. We keep all bialgebroid structure of $H$ over $A$ defined in the above and simplify its antipode to $T: H \rightarrow H$ defined by

$$
T(a \# h)=S\left(h^{(2)}\right) a^{(0)} \# S\left(h^{(1)}\right) a^{(1)}
$$

Proposition 2.1. With the above definition $(H, A, \alpha, \beta, \Delta, \epsilon, T)$ is a para Hopf algebroid.

Proof. We know it is a bialgebroid. We show that $T^{2}=i d$, and $T$ satisfies the condition (11). To show the former, since $T$ is an antialgebra map and for any $h \in \mathcal{H}$ we have $T^{2}(1 \# h)=1 \# S^{2}(h)$, it suffices to show $T^{2}(a \# 1)=a \# 1$ for any $a \in A$.

$$
\begin{aligned}
& T^{2}(a \# 1)=T\left(a^{(0)} \# a^{(1)}\right)=T\left(1 \# a^{(1)}\right) T(a \# 1)=\left(1 \# S\left(a^{(2)}\right)\right)\left(a^{(0)} \# a^{(1)}\right) \\
& =S\left(a^{(3)}\right) a^{(0)} \# S\left(a^{(2)}\right) a^{(1)}=S\left(a^{(1)}\right) a^{(0)} \# 1=a \# 1
\end{aligned}
$$

Now let us check the condition (11). It is shown that the condition (11) is multiplicative [14. So we may check it for $1 \# h$ and $a \# 1$ separately. We just check the latter and leave the other to the reader.

$$
\begin{aligned}
& T\left((a \# 1)^{(1)}\right)^{(1)}(a \# 1)^{(2)} \otimes_{A} T\left((a \# 1)^{(1)}\right)^{(2)} \\
& =\left(a^{(0)} \# a^{(1)}\right)^{(1)}(1 \# 1) \otimes_{A}\left(a^{(0)} \# 1\right)^{(2)}=\left(a^{(0)} \# a^{(1)}\right) \otimes_{A} 1 \# a^{(2)} \\
& =\beta\left(a^{(0)}\right)(1 \# 1) \otimes_{A} 1 \# a^{(1)}=1 \# 1 \otimes_{A} a^{(0)} \# a^{(1)}=1 \# 1 \otimes_{A} T(a \# 1)
\end{aligned}
$$

Example 2.3. Let $\mathcal{H}$ be a Hopf algebra with $S^{2}=i d$. Let $A=\mathcal{H}$ with the conjugation action, i.e., ha $=h^{(1)} a S\left(h^{(2)}\right)$. The right coaction that we propose is $\Delta_{A}(a)=a^{(2)} \otimes S\left(a^{(1)}\right)$. One can check that $A$ is a braided commutative algebra in $\mathcal{Y D}^{\mathcal{H}}$. So $A \# H$ is a para Hopf algebroid on $A$. 
Example 2.4. Let a discrete group $\Gamma$ acts from right by diffeomorphisms on a manifold $M$. One can check that $A=C^{\infty}(M)$ with trivial coaction of $\mathcal{H}=\mathbb{C} \Gamma$ satisfies the condition of the above proposition, hence $C^{\infty}(M) \rtimes \Gamma$ is a para Hopf algebroid over $C^{\infty}(M)$.

Let $\mathcal{C}$ and $\mathcal{D}$ be corings over $R$ and $S$ respectively. It is easy to check that $\mathcal{C} \otimes \mathcal{D}$ is a coring over $R \otimes S$, where $\mathcal{C} \otimes \mathcal{D}$ is $R \otimes S$ bimodule via $(r \otimes s)(c \otimes d)=r c \otimes s d$, and $(c \otimes d)(r \otimes s)=c r \otimes d s, c \in \mathcal{C}, d \in \mathcal{D}, r \in R$, and $s \in S$. Its coproduct and counit are defined by

$$
\begin{array}{ll}
\Delta: \mathcal{C} \otimes \mathcal{D} \rightarrow \mathcal{C} \otimes \mathcal{D} \otimes_{R \otimes S} \mathcal{C} \otimes \mathcal{D}, & \Delta(c \otimes d)=c^{(1)} \otimes d^{(1)} \otimes_{R \otimes S} c^{(2)} \otimes d^{(2)} \\
\epsilon: \mathcal{C} \otimes \mathcal{D} \rightarrow R \otimes S, & \epsilon(c \otimes d)=\epsilon(c) \otimes \epsilon(d) .
\end{array}
$$

On the other hand $\mathcal{C}^{\mathrm{cop}}$ which is $\mathcal{C}$ as a vector space has a coring structure over $R^{\text {op }}$ as follows. The $R^{\text {op }}$ bimodule structure of $\mathcal{C}^{\text {cop }}$ is defined in the usual way, i.e., $r_{1} c r_{2}:=r_{2} c r_{1}$. The coproduct and counit is defined by

$$
\begin{array}{lll}
\Delta: \mathcal{C}^{\text {cop }} \rightarrow \mathcal{C}^{\text {cop }} \otimes_{R^{\text {op }}} \mathcal{C}^{\text {cop }}, & & \Delta_{\mathcal{C}^{\text {cop }}}(c)=c^{(2)} \otimes_{R^{\text {op }}} c^{(1)} \\
\epsilon_{\mathcal{C}^{\text {cop }}}: \mathcal{C}^{\text {cop }} \rightarrow R^{\text {op }}, & \epsilon_{\mathcal{C}^{\text {cop }}}=\epsilon(c) .
\end{array}
$$

Now we can define enveloping coring of a coring $(\mathcal{C}, R)$, which is $\left(\mathcal{C} \otimes \mathcal{C}^{\text {cop }}, R \otimes\right.$ $\left.R^{\mathrm{op}}\right)$ defined as above. We denote this coring by $\left(\mathcal{C}^{\mathrm{e}}, R^{\mathrm{e}}\right)$.

\section{Hopf cyclic co/homology of corings}

In this section we recall the cyclic cohomology of coalgebras and then we will extend this theory to the category of corings. We will observe that the key concept that enables us to proceed is the notion of para Hopf algebroid.

Let $C$ be a coalgebra over a commutative ring $k$. Recall that the cocyclic module assigned to $C$ is $\left\{C^{n}=C^{n}(C)=C^{\otimes n+1}\right\}_{n \geq 0}$ with the following maps:

$$
\begin{aligned}
& \delta_{i}: C^{n} \rightarrow C^{n+1}, \quad i=0,1, \ldots, n+1 \\
& \delta_{i}\left(c_{0} \otimes c_{1} \otimes \ldots \otimes c_{n}\right)=\left(c_{0} \otimes c_{1} \otimes \ldots \otimes \Delta\left(c_{i}\right) \otimes \ldots \otimes c_{n}\right) \\
& \delta_{n+1}\left(c_{0} \otimes c_{1} \otimes \ldots \otimes c_{n}\right)=\left(c_{0}^{(2)} \otimes c_{1} \otimes \ldots \otimes c_{n} \otimes c_{0}^{(1)}\right) \\
& \sigma_{j}: C^{n} \rightarrow C^{n-1} j=0,1, \ldots, n-1 \\
& \sigma_{j}\left(c_{0} \otimes c_{1} \otimes \ldots \otimes c_{n}\right)=\left(c_{0} \otimes c_{1} \otimes \ldots \otimes \epsilon\left(c_{i+1}\right) \ldots \otimes c_{n}\right)
\end{aligned}
$$


$\tau: C^{n} \rightarrow C^{n}$

$\tau\left(c_{0} \otimes c_{1} \otimes \ldots \otimes c_{n}\right)=\left(c_{1} \otimes \ldots \otimes c_{n} \otimes c_{0}\right)$

Now let us try to extend the above structure for a coring $(\mathcal{C}, R)$. If we simply change the tensor over $k$ to tensor over $R$, the first problem that we face is that the cyclic operator $\tau$ and the last face $\delta_{n+1}$ are not well-defined. To fix this problem we observe that if we change the first tensor to tensor over $R^{e}$ and the others to tensor over $R$ then the operators are well-defined and we have the following proposition.

Proposition 3.1. Let $(\mathcal{C}, R)$ be a coring then the following define a cocyclic $k$-module.

$$
\begin{aligned}
& \quad\left\{C^{n}=C^{n}(\mathcal{C})=\mathcal{C} \otimes_{R^{e}} \mathcal{C}^{\otimes_{R}{ }^{n}}\right\}_{n \geq 1}, C^{0}=\mathcal{C} \otimes_{R^{e}} R \text { with the following maps: } \\
& \delta_{i}: C^{n} \rightarrow C^{n+1}, \quad i=0,1, \ldots, n+1 \\
& \delta_{i}\left(c_{0} \otimes_{R^{e}} c_{1} \otimes_{R} \ldots \otimes_{R} c_{n}\right)=\left(c_{0} \otimes_{R^{e}} c_{1} \otimes_{R} \ldots \otimes_{R} \Delta\left(c_{i}\right) \otimes_{R} \ldots \otimes_{R} c_{n}\right) \\
& \delta_{n+1}\left(c_{0} \otimes_{R^{e}} c_{1} \otimes_{R} \ldots \otimes_{R} c_{n}\right)=\left(c_{0}^{(2)} \otimes_{R^{e}} c_{1} \otimes_{R} \ldots \otimes_{R} c_{n} \otimes_{R} c_{0}^{(1)}\right) \\
& \sigma_{j}: C^{n} \rightarrow C^{n-1} j=0,1, \ldots, n-1 \\
& \sigma_{j}\left(c_{0} \otimes_{R^{e}} c_{1} \otimes_{R} \ldots \otimes_{R} c_{n}\right)=\left(c_{0} \otimes_{R^{e}} c_{1} \otimes_{R} \ldots \otimes_{R} \epsilon\left(c_{i+1}\right) \ldots \otimes_{R} c_{n}\right) \\
& \tau: C^{n} \rightarrow C^{n} \\
& \tau\left(c_{0} \otimes_{R^{e}} c_{1} \otimes_{R} \ldots \otimes_{R} c_{n}\right)=\left(c_{1} \otimes_{R^{e}} \ldots \otimes_{R} c_{n} \otimes_{R} c_{0}\right)
\end{aligned}
$$

Proof. See the proof of Theorem 3.1 as a more general case.

We denote its Hochschild, cyclic and periodic cyclic cohomology by $H H^{*}(\mathcal{C})$, $H C^{*}(\mathcal{C})$, and $H P^{*}(\mathcal{C})$ respectively.

Lemma 3.1. Let $(\mathcal{C}, R)$ be a $H$-coring module. Then for any $r, s \in R$, and $c \in \mathcal{C}$ we have

$$
\alpha(r) c \otimes_{H} s=c \otimes_{H} s r, \text { and } \beta(r) c \otimes_{H} s=c \otimes_{H} r s .
$$

Proof. Using the fact that $T^{2}=i d_{H}$ and $T \beta=\alpha$, we conclude that $T \alpha=\beta$. So $\alpha(r) c \otimes_{H} s=c \cdot \beta(r) \otimes_{H} s=c \otimes_{H} \beta(r) \cdot s=c \otimes_{H} \epsilon_{H}(\beta(r) \beta(s))=$ $c \otimes_{H} \epsilon_{H}(\beta(s r))=c \otimes_{H} s r$. And similarly, $\beta(r) c \otimes_{H} s=c \cdot \alpha(r) \otimes_{H} s=$ $c \otimes_{H} \alpha(r) \cdot s=c \otimes_{H} \epsilon_{H}(\alpha(r) \alpha(s))=c \otimes_{H} \epsilon_{H}(\alpha(r s))=c \otimes_{H} r s$.

Lemma 3.2. For any $n \geq 1, h \in H$, and $c_{1}, \ldots, c_{n} \in \mathcal{C}$ one has:

$$
\begin{aligned}
& T\left(h^{(1)}\right)^{(1)} h^{(2)} c_{1} \otimes_{R} \ldots \otimes_{R} T\left(h^{(1)}\right)^{(n-1)} h^{(n)} c_{n-1} \otimes_{R} T\left(h^{(1)}\right)^{(n)} c_{n}= \\
& c_{1} \otimes_{R} \ldots \otimes_{R} c_{n-1} \otimes_{R} T(h) c_{n}
\end{aligned}
$$


Proof. First, we see that the following defines a well-defined map,

$\Psi: H^{\otimes_{R} n} \otimes \mathcal{C}^{\otimes n} \rightarrow \mathcal{C}^{\otimes_{R} n}$

$\Psi\left(h_{1} \otimes_{R} \ldots \otimes_{R} h_{n} \otimes c_{1} \otimes \ldots \otimes c_{n}\right)=h_{1} c_{1} \otimes_{R} \ldots \otimes_{R} h_{n} c_{n}$.

Next, we apply $\Delta_{H}$, the comultiplication of $H, n-2$ times on the two sides of the crucial equation (11) to get

$T\left(h^{(1)}\right)^{(1)} h^{(2)} \otimes_{R} \ldots \otimes_{R} T\left(h^{(1)}\right)^{(n-1)} h^{(n)} \otimes_{R} T\left(h^{(1)}\right)^{(n)}=1 \otimes_{R} \ldots \otimes_{R} 1 \otimes_{R} T(h)$.

Then we apply both hand sides on $c_{1} \otimes_{R} \ldots \otimes_{R} c_{n}$.

The reader may ask what algebra except $R^{\mathrm{e}}$ can be used to balance the above complex to get a cocyclic module. This question is answered in the following theorem.

In the following theorem $\mathcal{C}$ is considered as a right $H$ module via the antipode of $H$ and $\mathcal{C}^{\otimes_{R}}$ is left $H$ module diagonally.

Theorem 3.1. Let $(H, R)$ be a para Hopf algebroid and $(\mathcal{C}, R)$ an $H$-module coring. Then the following define a cocyclic module:

$\left\{C^{n}=C^{n}(\mathcal{C})=\mathcal{C} \otimes_{H} \mathcal{C}^{\otimes_{R} n}\right\}_{n \geq 0}$ with the following maps:

$\delta_{0}, \delta_{1}: C^{0} \rightarrow C^{1}$, defined by,

$$
\begin{gathered}
\delta_{0}\left(c \otimes_{H} r\right)=c^{(1)} \otimes_{H} \beta(r) c^{(2)} \\
\delta_{1}\left(c \otimes_{H} r\right)=c^{(2)} \otimes_{H} \alpha(r) c^{(2)} \\
\delta_{i}: C^{n} \rightarrow C^{n+1}, \quad i=0,1, \ldots, n+1 \\
\delta_{i}\left(c_{0} \otimes_{H} c_{1} \otimes_{R} \ldots \otimes_{R} c_{n}\right)=\left(c_{0} \otimes_{H} c_{1} \otimes_{R} \ldots \otimes_{R} \Delta\left(c_{i}\right) \otimes_{R} \ldots \otimes_{R} c_{n}\right) \\
\delta_{n+1}\left(c_{0} \otimes_{H} c_{1} \otimes_{R} \ldots \otimes_{R} c_{n}\right)=\left(c_{0}^{(2)} \otimes_{H} c_{1} \otimes_{R} \ldots \otimes_{R} c_{n} \otimes_{R} c_{0}^{(1)}\right) \\
\sigma_{j}: C^{n} \rightarrow C^{n-1} j=0,1, \ldots, n-1 \\
\sigma_{j}\left(c_{0} \otimes_{H} c_{1} \otimes_{R} \ldots \otimes_{R} c_{n}\right)=\left(c_{0} \otimes_{H} c_{1} \otimes_{R} \ldots \otimes_{R} \epsilon\left(c_{i+1}\right) \otimes_{R} \ldots \otimes_{R} c_{n}\right) \\
\left.\tau: C^{n} \rightarrow C^{n} \quad \otimes_{R}{ }^{\prime} \ldots \otimes_{R} c_{n} \otimes_{R} c_{0}\right)
\end{gathered}
$$


Proof. We should show that $\delta_{i}, \sigma_{j}$, and $\tau$ satisfy the cocyclic module relations.

First cosimplicial module relations:

$$
\begin{gathered}
\delta_{i} \delta_{j}=\delta_{j} \delta_{i-1} j<i, \\
\sigma_{i} \sigma_{j}=\sigma_{j} \sigma_{i+1} j \leq i, \\
\sigma_{j} \delta_{i}=\left\{\begin{array}{cc}
\delta_{i} \sigma_{j-1} & \text { if } i<j \\
i d & \text { if } i=j \text { or } i=j+1 \\
\delta_{i-1} \sigma_{j} & \text { if } i>j+1,
\end{array}\right.
\end{gathered}
$$

and relations of cyclic operator with faces and degeneracies: $\tau \delta_{i}=\delta_{i-1} \tau$, $\tau \sigma_{j}=\sigma_{j-1} \tau$ and the crucial relation $\tau^{n+1}=i d$.

We leave to the reader to check the the above relations because they are the same as the cyclic structure of coalgebras but we show the above maps are well-defined. It is easy to see that $\delta_{i}, i=0, \ldots, n$ and $\sigma_{j}, j=0,1, \ldots, n-1$ are well-defined due to the fact that $\Delta_{\mathcal{C}}$ and $\epsilon_{\mathcal{C}}$ are $H$-linear. If we prove $\tau$ is well-defined, then since $\delta_{n+1}=\tau \delta_{0}$ we have done this stage completely.

In degree zero the cyclic operator is $i d_{C^{0}}$ and hence it is well-defined. Let $n \geq 1, h \in H, r \in R$ and $c_{0}, \ldots, c_{n} \in \mathcal{C}$.

Using Lemma 3.2 we have

$$
\begin{aligned}
& \tau\left(c_{0} \otimes h \cdot c_{1} \otimes \ldots \otimes c_{n}\right)=\tau\left(c_{0} \otimes h^{(1)} c_{1} \otimes \ldots \otimes h^{(n)} c_{n}\right) \\
& =h^{(1)} c_{1} \otimes_{H} h^{(2)} c_{2} \otimes_{R} \ldots \otimes_{R} h^{(n)} c_{n} \otimes_{R} c_{0} \\
& =c_{1} T\left(h^{(1)}\right) \otimes_{H} h^{(2)} c_{2} \otimes_{R} \ldots \otimes_{R} h^{(n)} c_{n} \otimes_{R} c_{0} \\
& =c_{1} \otimes_{H} T\left(h^{(1)}\right)^{(1)} h^{(2)} c_{2} \otimes_{R} \ldots \otimes_{R} T\left(h^{(1)}\right)^{(n-1)} h^{(n)} c_{n} \otimes_{R} T\left(h^{(1)}\right)^{(n)} c_{0} \\
& =c_{1} \otimes_{H} c_{2} \otimes_{R} \ldots \otimes_{R} c_{n} \otimes_{R} T(h) c_{0}=\tau\left(c_{0} h \otimes c_{1} \ldots \otimes c_{n}\right) .
\end{aligned}
$$

There are also some ambiguities over $\otimes_{R}$ 's which we should get rid of. We show $\tau$ is well-defined over the first $\otimes_{R}$ and leave the rest to the reader.

$$
\begin{aligned}
& \tau\left(c_{0} \otimes c_{1} \cdot r \otimes \ldots \otimes c_{n}\right)= \\
& \beta(r) c_{1} \otimes_{H} c_{2} \otimes_{R} \ldots \otimes_{R} c_{n} \otimes_{R} c_{0}= \\
& c_{1} \cdot \alpha(r) \otimes_{H} c_{2} \otimes_{R} \ldots \otimes_{R} c_{n} \otimes_{R} c_{0}= \\
& c_{1} \otimes_{H} \alpha(r) \triangleright c_{2} \otimes_{R} \ldots \otimes_{R} c_{n} \otimes_{R} c_{0}= \\
& c_{1} \otimes_{H} \alpha(r) c_{2} \otimes_{R} \ldots \otimes_{R} c_{n} \otimes_{R} c_{0}= \\
& \tau\left(c_{0} \otimes c_{1} \otimes r \cdot c_{2} \otimes \ldots \otimes c_{n}\right) .
\end{aligned}
$$


The Hopf cyclic cohomology of $(\mathcal{C}, R)$ under the action of $H$ is denoted by $H C_{H}^{*}(\mathcal{C}, R)$.

Definition 3.1. We say a $H$-module coring $(\mathcal{C}, R)$ is $H$ coseparable if there is a $H$-linear map $\delta: \mathcal{C} \otimes_{R} \mathcal{C} \rightarrow R$ satisfying

1. $\delta \Delta=\epsilon$

2. $\left(i d_{\mathcal{C}} \otimes_{R} \delta\right)\left(\Delta \otimes_{R} i d_{\mathcal{C}}\right)=\left(\delta \otimes_{R} i d_{\mathcal{C}}\right)\left(i d_{\mathcal{C}} \otimes_{R} \Delta\right)$

For example if an algebra extension $B \hookrightarrow A$ is split, i.e., there is a $B-B$ bimodule map $E: A \rightarrow B$ such that $E(1)=1$, then the Sweedler coring $A \otimes_{B} A$ is coseparable [10].

Definition 3.2. (Haar system for bialgebroids) Let $(H, R)$ be a bialgebroid. Let $\theta: H \longrightarrow R$ be a right R-module map. We call $\theta$ a left Haar system for $H$ if for all $h \in H$

$$
\sum \alpha\left(\theta\left(h^{(1)}\right)\right) h^{(2)}=\beta(\theta(h)) 1_{H} .
$$

We call $\theta$ a normal left Haar system if $\theta\left(1_{H}\right)=1_{R}$.

Proposition 3.2. If a para Hopf algebroid $H$ is coseparable as a module coring over itself, then it admits a normalized Haar system.

Proof. Let $\delta: H \otimes_{R} H \rightarrow R$ be a coseparating map for $H$. We define $\theta: H \rightarrow R$ by $\theta(h)=\delta(1, h)$. We see that $\theta\left(h^{(1)}\right) \otimes_{R} h^{(2)}=\delta\left(1, h^{(1)}\right) \otimes_{R} h^{(2)}=$ $1 \otimes_{R} \delta\left(1 \otimes_{R} h\right)=1 \otimes_{R} \theta(h)$. So $\theta$ is a Haar system. It is easily seen that normality comes from the first condition of coseparability.

Proposition 3.3. (14]) Let $(H, R)$ be a para Hopf algebroid that admits a normal left Haar system. Then $H C_{H}^{2 i+1}(H, R)=0$ and $H C_{H}^{2 i}(H, R)=$ $\operatorname{ker}(\alpha-\beta)$ for all $i \geq 0$.

Proposition 3.4. If $(\mathcal{C}, R)$ is $H$ coseparable, then

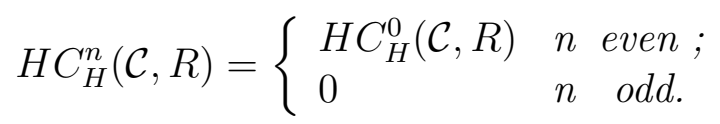

Proof. It is easily checked that the following is a homotopy between identity and zero map on the Hochschild complex. The rest is a standard application of Connes $S I B$ long exact sequence.

$$
\begin{aligned}
& h: C^{n} \rightarrow C^{n-1} \\
& h\left(c_{0} \otimes_{H} c_{1} \otimes_{R} \ldots \otimes_{R} c_{n}\right)=c_{0}^{(1)} \otimes_{H} \delta\left(c_{0}^{(2)} \otimes_{R} c_{1}\right) \otimes_{R} c_{2} \otimes_{R} \ldots \otimes_{R} c_{n}
\end{aligned}
$$


As we know corings generalize coalgebras and algebras simultaneously, this interestingly happens for their cyclic co/homology too. Obviously this generalization can be seen for coalgebras but for algebras we need first to see how the dual of the above cocyclic module looks like. We recall from [5] see also [13], roughly speaking, dual of a cocyclic module, say $C^{n}$, is a cyclic module with the same vector space structure whose faces are degeneracies of $C^{n}$ plus the extra degeneracy which is defined by $\sigma_{-1}=\tau \sigma_{n-1}$. The degeneracies of the dual cyclic module are faces of the original cocyclic module, and finally the cyclic operator of the dual cyclic module is the inverse of the cyclic operator of the original cyclic module. In the following we denote the dual of the above cocyclic module by $\hat{C}_{n}(\mathcal{C})$. Its cyclic structure is given by: $d_{0}, d_{1}: \hat{C}_{1} \rightarrow \hat{C}_{0}$ defined by

$d_{0}\left(c_{0} \otimes_{H} c_{1}\right)=c_{0} \otimes_{H} \epsilon\left(c_{1}\right), d_{1}\left(c_{0} \otimes_{H} c_{1}\right)=c_{1} \otimes_{H} \epsilon\left(c_{0}\right)$

$d_{i}: \hat{C}_{n} \rightarrow \hat{C}_{n-1}, \quad i=0,1, \ldots, n$

$d_{i}\left(c_{0} \otimes_{H} c_{1} \otimes_{R} \ldots \otimes_{R} c_{n}\right)=\left(c_{0} \otimes_{H} c_{1} \otimes_{R} \ldots \otimes_{R} \epsilon\left(c_{i+1}\right) \otimes_{R} \ldots \otimes_{R} c_{n}\right)$

$d_{n}\left(c_{0} \otimes_{H} c_{1} \otimes_{R} \ldots \otimes_{R} c_{n}\right)=\left(c_{n} \otimes_{H} \epsilon\left(c_{0}\right) \otimes_{R} \ldots \otimes_{R} c_{n-1}\right)$

$s_{j}: \hat{\mathcal{C}}_{n} \rightarrow \hat{C}_{n+1} \quad j=0,1, \ldots, n$

$s_{n}\left(c_{0} \otimes_{H} c_{1} \otimes_{R} \ldots \otimes_{R} c_{n}\right)=\left(c_{0} \otimes_{H} c_{1} \otimes_{R} \ldots \otimes_{R} \Delta\left(c_{i+1}\right) \ldots \otimes_{R} c_{n}\right)$

$s_{j}\left(c_{0} \otimes_{H} c_{1} \otimes_{R} \ldots \otimes_{R} c_{n}\right)=\left(c_{0}^{(2)} \otimes_{H} c_{1} \otimes_{R} \ldots \otimes_{R} \epsilon\left(c_{i+1}\right) \ldots \otimes_{R} c_{n} \otimes_{R} c_{0}^{(1)}\right)$

$t: \hat{C}_{n} \rightarrow \hat{C}_{n}$

$t\left(c_{0} \otimes_{H} c_{1} \otimes_{R} \ldots \otimes_{R} c_{n}\right)=\left(c_{n} \otimes_{H} c_{0} \otimes_{R} c_{1} \otimes_{R} \ldots \otimes_{R} c_{n}\right)$

Proposition 3.5. Cyclic module of an algebra $A$ is isomorphic to dual Hopfcyclic module of the Sweedler coring $A \otimes A^{o p}$ as $A \otimes A^{o p}$ - module coring.

Proof. Let $C_{n}(A)$ denote the ordinary cyclic complex of the algebra $A$. We define the following maps and leave to the reader to check that these map are cyclic maps, i.e. they commute with cyclic structure, and are inverse of each other.

$$
\begin{aligned}
& \Psi: C_{n}(A) \longrightarrow \hat{C}_{n}\left(A^{\mathrm{e}}\right) \\
& \Psi\left(a_{0} \otimes \ldots \otimes a_{n}\right)=1_{A^{\mathrm{e}}} \otimes_{A^{\mathrm{e}}} a_{0} \otimes 1_{A} \otimes_{R} \ldots \otimes_{R} a_{n-1} \otimes 1_{A} \otimes_{R} a_{n-1} \otimes a_{n} \\
& \quad \Phi: \hat{C}_{n}\left(A^{\mathrm{e}}\right) \longrightarrow C_{n}(A) \\
& \Phi\left(a_{0} \otimes b_{0} \otimes_{A^{\mathrm{e}}} a_{1} \otimes b_{1} \otimes_{R} \ldots \otimes_{R} a_{n} \otimes b_{n}\right)=b_{0} a_{1} \otimes b_{1} a_{2} \otimes \ldots \otimes b_{n-1} a_{n} \otimes b_{n} a_{0}
\end{aligned}
$$


Any para Hopf algebroid is a module coring on itself via multiplication. We show that cocyclic module of a para Hopf algebroid defined in [14] is isomorphic to its cocyclic module as a module coring on itself. To this end, we first recall cocyclic module of a para Hopf algebroid from [14.

Let $(H, R)$ be para Hopf algebroid. Its cocyclic module as a para Hopf algebroid, also called Connes-Moscovici cocyclic module of $H$ is defined as follows:

$$
H_{\natural}^{0}=R \text {, and } H_{\natural}^{n}=H \otimes_{R} H \otimes_{R} \cdots \otimes_{R} H \quad \text { ( } n \text {-fold tensor product). }
$$

The cofaces $\delta_{i}$ and codegeneracies $\sigma_{i}$ are defined by:

$$
\begin{aligned}
\delta_{0}(a)=\alpha(a), \delta_{1}(a)=\beta(a) & \text { for all } a \in R=H_{\natural}^{0} \\
\delta_{0}\left(h_{1} \otimes_{R} \cdots \otimes_{R} h_{n}\right)= & 1_{H} \otimes_{R} h_{1} \otimes_{R} \cdots \otimes_{R} h_{n} \\
\delta_{i}\left(h_{1} \otimes_{R} \cdots \otimes_{R} h_{n}\right)= & h_{1} \otimes_{R} \cdots \otimes_{R} \Delta\left(h_{i}\right) \otimes_{R} \cdots \otimes_{R} h_{n} \text { for } 1 \leq i \leq n \\
\delta_{n+1}\left(h_{1} \otimes_{R} \cdots \otimes_{R} h_{n}\right) & =h_{1} \otimes_{R} \cdots \otimes_{R} h_{m} \otimes_{R} 1_{H} \\
\sigma_{i}\left(h_{1} \otimes_{R} \cdots \otimes_{R} h_{n}\right) & =h_{1} \otimes_{R} \cdots \otimes_{R} \epsilon\left(h_{i+1}\right) \otimes_{R} \cdots \otimes_{R} h_{n} \text { for } 0 \leq i \leq n
\end{aligned}
$$

The cyclic operator $\tau$ defined by

$$
\tau_{n}\left(h_{1} \otimes_{R} \cdots \otimes_{R} h_{n}\right)=T\left(h_{1}\right) \triangleright\left(h_{2} \otimes_{R} \ldots \otimes_{R} h_{n} \otimes_{R} 1_{H}\right),
$$

Proposition 3.6. Let $(H, R)$ be a para Hopf algebroid. Then its Hopf-cyclic module as module coring over itself is isomorphic to its Connes-Moscovici cocyclic module.

Proof. We define the following maps and leave to the reader to verify that they are cyclic maps and inverse to one another.

$\Psi: C^{n}(H) \longrightarrow H_{\natural}^{n}$

$\Psi\left(h_{0} \otimes_{H} h_{1} \otimes_{R} \ldots \otimes_{R} h_{n}\right)=T\left(h_{0}\right) \triangleright\left(h_{1} \otimes_{R} \ldots \otimes_{R} h_{n}\right)$

$\Phi: H_{\natural}^{n} \longrightarrow C^{n}(H)$

$\Phi\left(h_{1} \otimes_{R} \ldots \otimes_{R} h_{n}\right)=1_{H} \otimes_{H} h_{1} \otimes_{R} \ldots \otimes_{R} h_{n}$. 


\section{References}

[1] T. Brzezinski, and R. Wisbauer, Corings and comodules. London Mathematical Society Lecture Note Series, 309. Cambridge University Press, Cambridge, 2003.

[2] T. Brzezinski, G. Militaru, Bialgebroids, $\times_{A}$-bialgebras and duality. J. Algebra 251, 279-294 (2002).

[3] A. Cannas da Silva and A. Weinstein, Geometric models for noncommutative algebras. Berkeley Mathematics Lecture Notes, 10. American Mathematical Society, Providence, RI; Berkeley Center for Pure and Applied Mathematics, Berkeley, CA, 1999.

[4] A.Connes and H. Moscovici, Cyclic cohomology and Hopf algebra symmetry. Conference Mosh Flato 1999 (Dijon). Lett. Math. Phys. 52 (2000), no. $1,1-28$.

[5] A. Connes, Noncommutative differential geometry. Inst. Hautes tudes Sci. Publ. Math. No. 62 (1985), 257-360

[6] Connes, A., Cohomologie cyclique et foncteurs Ext ${ }^{n}$. C. R. Acad. Sci. Paris Ser. I Math. 296 (1983), No. 23, 953-958.

[7] A. Connes and H. Moscovici, Differential cyclic cohomology and Hopf algebraic structures in transverse geometry. Essays on geometry and related topics, Vol. 1, 2, 217-255, Monogr. Enseign. Math., 38, Enseignement Math., Geneva, 2001.

[8] A. Connes and H. Moscovici, Hopf algebras, Cyclic Cohomology and the transverse index theorem., Comm. Math. Phys. 198 (1998), no. 1, 199246.

[9] D. Etingof and D. Nikshych, Dynamical Quantum Groups at roots of 1. arXiv:math.QA/0003221 30 Mar 2000.

[10] F. Guzmn, Cointegrations, relative cohomology for comodules, and coseparable corings. J. Algebra 126 (1989), no. 1, 211-224.

[11] P. M. Hajac, M. Khalkhali, B. Rangipour, and Y. Sommerhäuser, Hopfcyclic homology and cohomology with coefficients. C. R. Math. Acad. Sci. Paris 338 (2004), no. 9, 667-672. 
[12] J. Huebschmann, Poisson cohomology and quantization. J. Reine Angew. Math. 408 (1990), 57-113.

[13] M. Khalkhali and B. Rangipour, A note on cyclic duality and Hopf algebras, Communications in Algebra, 33 (2005), no 3. 763-773.

[14] M. Khalkhali, and B. Rangipour Para-Hopf algebroids and their cyclic cohomology. Lett. Math. Phys. 70 (2004), no. 3, 259-272.

[15] M. Khalkhali, and B. Rangipour, Invariant cyclic homology. K-Theory 28(2), 183-205, 2003.

[16] M. Khalkhali, and B. Rangipour A New Cyclic Module for Hopf Algebras. $K$-Theory 27 (2002), no. 2, 111-131.

[17] J. L. Loday, Cyclic Homology. Springer-Verlag, (1992).

[18] J. H. Lu, Hopf algebroids and quantum groupoids. Internat. J. Math. 7 (1996), no. 1, 47-70.

[19] G. Maltsiniotis, Groupoids quantiques. C.R. Acad. Sci. Paris, 314 (1992), 249-252.

[20] J. Mrčun, The Hopf algebroids of functions on étale groupoids and their principal Morita equivalence. J. Pure Appl. Algebra 160 (2001), no. 2-3, 249-262.

[21] D. C. Ravenel, Complex cobordism and stable homotopy groups of spheres. Pure and Applied Mathematics, 121. Academic Press Inc., Orlando, FL, 1986.

[22] G. Rinehart, Differential forms on general commutative algebras. Trans. Amer. Math. Soc. 108 1963, 195-222.

[23] P. Xu, Quantum groupoids. Comm. Math. Phys. 216 (2001), no. 3, 539581. 